\title{
Robust Leaderless Consensus of Uncertain Multiagent Systems with Fast Switching Topologies
}

\author{
Wei Liu, Shaolei Zhou, Shi Yan, and Gaoyang Yin \\ Department of Control Engineering, Naval Aeronautical and Astronautical University, Yantai 264001, China \\ Correspondence should be addressed to Wei Liu; weiliu.sd.china@hotmail.com
}

Received 18 June 2015; Revised 28 August 2015; Accepted 12 October 2015

Academic Editor: Yuan Fan

Copyright (C) 2015 Wei Liu et al. This is an open access article distributed under the Creative Commons Attribution License, which permits unrestricted use, distribution, and reproduction in any medium, provided the original work is properly cited.

\begin{abstract}
This paper investigates the robust leaderless consensus problem of uncertain multiagent systems with directed fast switching topologies. The topologies are assumed to jointly contain a directed spanning tree. Based on a special property of the graph Laplacian matrix, the consensus problem is converted into a stabilization problem by performing a proper variable transformation. Averaging method is employed for analysis. It is proved that if the topologies switch sufficiently fast and the controllers are properly designed, the robust leaderless consensus can still be achieved even when all the possible topologies are unconnected in the switching time intervals. Finally, a numerical simulation is provided to illustrate the effectiveness of the theoretical results.
\end{abstract}

\section{Introduction}

In the past few years, the consensus problem of multiagent systems has drawn great attention for its broad potential applications in many areas such as cooperative control of vehicle, unmanned air vehicle formation, and flocking control [1-3]. Consensus means that all agents will reach a common state in a cooperative fashion throughout distributed controllers. It is called leaderless consensus if there is no specified leader in the multiagent systems. Since agents in the multiagent systems are coupled through networks, the consensus achievement depends on not only the individual agent dynamic but also the structure of the interaction topology.

In many applications, the interaction topology among agents may change dynamically. This may happen when the communication links among agents may be unreliable due to disturbance or subject to communication range limitations [4]. Motivated by this observation, many efforts have been made to investigate this topic. A pioneer work was shown in [4], in which it was proved that the consensus of first order multiagent systems could be achieved if the topologies jointly contained a directed spanning tree. Some conclusions of the first and second order dynamics were obtained in [5-8]. The consensus problem of a class of heterogeneous multiagent systems including first and second order dynamics was solved in [9]. Note that the consensus problem of first or second order dynamics is mainly solved using the stochastic indecomposable aperiodic (SIA) based approach which is not applicable to the higher order dynamics case. Thus, several other approaches are employed to solve the consensus problem for higher order multiagent systems with different topology conditions. In [10], under undirected jointly connected communication topologies, the leaderless consensus problem of high-order linear multiagent systems was addressed using extended Barbalat's lemma. When each possible directed topology was strongly connected and balanced [11], leaderless consensus problem of linear multiagent systems was solved with common Lyapunov function approach. Using multiple Lyapunov function approach, the leader-following [12] and leaderless [13] consensus problems of linear multiagent systems were addressed with the assumption that each possible topology contained a directed spanning tree. Based on averaging method, the leader-following consensus problems of linear multiagent systems with jointly connected topologies were solved with and without parameter uncertainties in [14] and [15], respectively. Under undirected topologies [16], the leader-following consensus problem of nonlinear multiagent systems was investigated with jointly connected topologies. Then, the results were extended to the case with distributed 
adaptive protocols [17]. In [18], the leaderless consensus problem of a certain type of nonlinear system was investigated with fast bidirectional switching topologies.

In contrast with the leader-following consensus problem, in the homogeneous multiagent systems, the leaderless consensus problem which can include the leader-following consensus problem as a special case is more complex and challenging especially with directed communication topologies. Since the leader-following consensus problem can be conveniently converted into a stabilization problem by constructing tracking error variables, the presence of the leader in the multiagent systems facilitates the derivation [13]. As for the leaderless consensus problem, there is no specified leader. Furthermore, when the topology is assumed to be undirected, the graph Laplacian matrix is positive semidefinite. This property facilitates the construction of the Lyapunov function greatly in the derivation. As for directed topologies, the graph Laplacian matrices are not necessarily symmetric. This increases the analysis difficulty especially when there are other specified constraints such as uncertainties in systems dynamics or switching communication topologies.

Motivated by this, the objective of this paper is to solve the robust leaderless consensus problem of uncertain multiagent systems with directed fast switching topologies. It is assumed that the switching topologies jointly contain a directed spanning tree. The analysis process is of two steps. Firstly, based on the property that the graph Laplacian matrix can be factored into the product of two specific matrices, the consensus problem with switching topologies is converted into a stabilization problem of a switched system by constructing a proper disagreement vector. Secondly, by using averaging method which is widely used for stability analysis of fast switching systems, sufficient conditions for achieving the leaderless consensus are obtained. It is shown that if the topologies switch sufficiently fast and the feedback gain matrices in the consensus controllers are properly designed, consensus can still be achieved even when the topologies are not connected in the switching time intervals. The main contribution of our paper is that the leaderless consensus problem of high-order dimension multiagent systems is solved under fast switching topologies which jointly contain a directed spanning tree.

The remainder of this paper is organized as follows. In Section 2, some preliminaries are provided. In Section 3, the robust leaderless consensus problem is solved with fast switching topologies. In Section 4, a simulation example is presented. Section 5 is the conclusion.

\section{Preliminaries}

2.1. Notations. Throughout this paper, the following notations will be used. $\mathbb{R}^{n \times n}$ and $\mathbb{C}^{n \times n}$ denote the set of $n \times n$ real and complex matrices, respectively. $\otimes$ denotes the Kronecker product. For $\mu \in \mathbb{C}$, the real part is $\operatorname{Re}(\mu)$. $I_{n}$ is the $n \times n$ identity matrix. For a square matrix $A, \lambda(A)$ denotes the eigenvalues of matrix $A ; A>B(A \geq B)$ means that $A-B$ is positive definite (resp., positive semidefinite). For a symmetrical matrix $A, \max \{\lambda(A)\}(\min \{\lambda(A)\})$ denotes the largest (smallest) eigenvalue of $A$.
2.2. Graph Theory. A directed graph $\mathscr{G}=(\mathscr{V}, \mathscr{E}, \mathscr{A})$ contains the vertex set $\mathscr{V}=\{1,2, \ldots, N\}$, the directed edges set $\mathscr{E} \subseteq$ $\mathscr{V} \times \mathscr{V}$, and the weighted adjacency matrix $\mathscr{A}=\left[a_{i j}\right]_{N \times N}$ with nonnegative elements $a_{i j} . a_{i j}>0$ if there is a directed edge between vertices $i$ and $j ; a_{i j}=0$ otherwise. The set of neighbors of $i$ is defined as $\mathscr{N}_{i}:=\left\{j \in \mathscr{V}: a_{i j}=1\right\}$. A directed path is a sequence of ordered edges of the form $\left(i_{1}, i_{2}\right),\left(i_{2}, i_{3}\right), \ldots$, where $i_{j} \in \mathscr{V}$. The Laplacian matrix of the topology $\mathscr{G}$ is defined as $\mathscr{L}=\left[\mathscr{L}_{i j}\right]_{N \times N}$, where $\mathscr{L}_{i i}=\sum_{j \neq i} a_{i j}$ and $\mathscr{L}_{i j}=-a_{i j}$. Then, 0 is an eigenvalue of $\mathscr{L}$ with $1_{N}$ as the eigenvector. A directed graph is said to have a spanning tree if there is a vertex called the root such that there is a directed path from this vertex to every other vertex.

In this paper, the communication topology is molded by a directed graph and we assume that the communication topology is time-varying. Denote $\widehat{\mathscr{G}}=\left\{\mathscr{G}_{1}, \mathscr{G}_{2}, \ldots, \mathscr{G}_{m}\right\}$, $m \geq 1$, to be the set of all possible directed topologies. We define the switching signal $\sigma(t)$, where $\sigma(t):[0,+\infty) \rightarrow$ $\mathscr{P}=\{1,2, \ldots, p\} .0=t_{0}<t_{1}<t_{2}<\cdots$ denote the switching instants of $\sigma(t)$. Let $\mathscr{G}_{\sigma(t)} \in \widehat{\mathscr{G}}$ be the communication topology at time $t$. Across each time interval $\left[t_{j}, t_{j+1}\right), j \in \mathbb{Z}$, the graph $\mathscr{G}_{\sigma(t)}$ is fixed.

A union graph of a collection of graphs $\mathscr{G}_{1}, \ldots, \mathscr{G}_{m}$ is defined as a graph, denoted by $\overline{\mathscr{G}}_{\sigma(t)}$ with the same vertex set $\mathscr{V}$ and the edge set equaling the union of the edge sets of all the graphs in the collection, and connection weight between edges $i$ and $j$ is the sum of $a_{i j}$ of $\mathscr{G}_{1}, \ldots, \mathscr{G}_{m}$. The collection $\mathscr{G}_{1}, \ldots, \mathscr{G}_{m}$ jointly contains a directed spanning tree if its union graph $\overline{\mathscr{G}}_{\sigma(t)}$ contains a directed spanning tree. Let $\overline{\mathscr{L}}_{\sigma(t)}$ be the Laplacian matrix of $\overline{\mathscr{G}}_{\sigma(t)}$.

Lemma 1 (see [4]). Zero is a simple eigenvalue of $\mathscr{L}$ and all the other nonzero eigenvalues have positive real parts if and only if the graph $\mathscr{G}$ has a directed spanning tree.

\section{Problem Formulation and Main Results}

Consider a multiagent system composed of $N$ agents with the following uncertain dynamics:

$$
\dot{x}_{i}(t)=(A+\Delta A) x_{i}(t)+B u_{i}(t), \quad i=1,2, \ldots, N,
$$

where $x_{i}(t) \in \mathbb{R}^{n}, u_{i}(t) \in \mathbb{R}^{p}$ are the state and the control input of the $i$ th agent, respectively. $A$ and $B$ are constant system matrices with compatible dimensions. $\Delta A$ represents the time-varying parameter uncertainty and is assumed to be in the form of $\Delta A=D F(t) H$, where $D$ and $H$ are known matrices of appropriate dimensions which characterize the structure of the uncertainty. $F(t)$ is an uncertain matrix satisfying $F(t)^{T} F(t) \leq \rho^{2} I$ and $\rho>0$ is a given constant.

Definition 2. The consensus of system (1) is said to be achieved with any finite initial value $x_{i}(0)$, if there exists a controller $u_{i}(t)$ such that

$$
\lim _{t \rightarrow \infty}\left\|x_{i}(t)-x_{j}(t)\right\|=0, \quad \forall i, j=1,2, \ldots, N
$$


In order to achieve consensus, the following distributed consensus controller based on local relative states information of neighbor agents is proposed:

$$
u_{i}(t)=c K \sum_{j=1}^{N} a_{i j}^{\sigma(t)}(t)\left(x_{j}(t)-x_{i}(t)\right), \quad i=1, \ldots, N,
$$

where $K \in \mathbb{R}^{p \times n}$ is the feedback matrix to be designed, $c$ is the coupling strength to be selected, and $a_{i j}^{\sigma(t)}(t)$ is the element of the adjacency matrix $\mathscr{A}_{\sigma(t)}$ of the graph $\mathscr{G}_{\sigma(t)}$.

The closed-loop system dynamics of (1) with the controller (3) is

$$
\dot{x}(t)=\left(I_{N} \otimes(A+\Delta A)-c \mathscr{L}_{\sigma(t)} \otimes B K\right) x(t),
$$

where $x(t)=\left[x_{1}(t)^{T}, x_{2}(t)^{T}, \ldots, x_{N}(t)^{T}\right]^{T}$ and $\mathscr{L}_{\sigma(t)} \in \mathbb{R}^{N \times N}$ is the Laplacian matrix of the graph $\mathscr{G}_{\sigma(t)}$.

Without loss of generality, consider an infinite sequence of nonempty, bounded, nonoverlapping, and contiguous time intervals $\left[t_{k}, t_{k+1}\right), k=0,1, \ldots$, with $t_{0}=0, t_{k+1}-t_{k} \leq T$ for some constant $T>0$. Suppose that, in each interval $\left[t_{k}, t_{k+1}\right)$, there is a sequence of $m_{k}$ nonoverlapping subintervals

$$
\begin{aligned}
{\left[t_{k}^{1}, t_{k}^{2}\right), \ldots,\left[t_{k}^{j}, t_{k}^{j+1}\right), \ldots,\left[t_{k}^{m_{k}}, t_{k}^{m_{k}+1}\right), } & \\
t_{k} & =t_{k}^{1}, t_{k+1}=t_{k}^{m_{k}+1},
\end{aligned}
$$

satisfying $t_{k}^{j+1}-t_{k}^{j} \geq \tau, 1 \leq j \leq m_{k}$ for a given constant $\tau>0$, such that, during each of such subintervals, the communication topology is fixed. The graph of communication topology is denoted by $\mathscr{G}_{k_{j}}$. In each time interval $\left[t_{k}^{j}, t_{k}^{j+1}\right)$, the topology is not necessarily connected.

Assumption 3. In this paper, we assume that across each time interval $\left[t_{k}, t_{k+1}\right), k=0,1, \ldots$, the union graph $\overline{\mathscr{G}}$ contains a directed spanning tree and remains the same.

Lemma 4 (see [19]). For any given $x, y \in \mathbb{R}^{n}$ and matrices $P>0, D$, and $S$ of appropriate dimensions, one has

$$
2 x^{T} D S y \leq x^{T} D P D^{T} x+y^{T} S^{T} P^{-1} S y .
$$

Lemma 5 (see [20]). For a Laplacian matrix $\mathscr{L} \in \mathbb{R}^{N \times N}$ of graph $\mathscr{G}$ and a full row rank matrix $E \in \mathbb{R}^{(N-1) \times N}$ defined as

$$
E=\left[\begin{array}{ccccc}
1 & -1 & 0 & \cdots & 0 \\
0 & 1 & -1 & \cdots & 0 \\
\vdots & \vdots & \ddots & \vdots & \vdots \\
0 & 0 & 0 & 1 & -1
\end{array}\right],
$$

there exists a matrix $M \in \mathbb{R}^{N \times(N-1)}$ such that $\mathscr{L}=M E$. Furthermore, if the graph has a directed spanning tree, $M$ is full column rank and $\operatorname{Re}(\lambda(E M))>0$.

Remark 6. From the definition of union graph $\overline{\mathscr{G}}$ of a collection of graphs $\left\{\mathscr{G}_{1}, \ldots, \mathscr{G}_{m}\right\}$, we can obtain that the
Laplacian matrix $\overline{\mathscr{L}}$ of $\overline{\mathscr{G}}$ is the sum of the Laplacian matrices $\left\{\mathscr{L}_{1}, \ldots, \mathscr{L}_{m}\right\}$ of $\left\{\mathscr{G}_{1}, \ldots, \mathscr{G}_{m}\right\}$; that is, $\overline{\mathscr{L}}=\mathscr{L}_{1}+\cdots+\mathscr{L}_{m}$. Since $\overline{\mathscr{G}}$ contains a directed spanning tree, $\overline{\mathscr{L}}$ has the property shown in Lemma 5 and so is $\overline{\mathscr{L}}^{\text {av }}=\sum_{i=1}^{N} \omega_{i} \mathscr{L}_{i}$, where $\omega_{i}>0$ and $\sum_{i=1}^{N} \omega_{i}=1$.

Lemma 7 (see [20]). If the graph fulfills Assumption 3, there exist a positive definite matrix $Q$ and a positive scalar $\alpha$ such that

$$
(E M)^{T} Q+Q E M>\alpha Q,
$$

where $0<\alpha<2 \min \{\operatorname{Re}(\lambda(E M))\}, E$ is defined in (7), and $M$ is a matrix satisfying $\mathscr{L}=M E$.

Let $\xi(t)=\left(E \otimes I_{n}\right) x(t)$, where $\xi_{i}(t)=x_{i}(t)-x_{i+1}(t), i=$ $1,2, \ldots, N-1$. Using Lemma 5 , closed-loop system dynamics (4) can be rewritten as

$$
\dot{\xi}(t)=\left(I_{N-1} \otimes(A+\Delta A)-c E M_{\sigma(t)} \otimes B K\right) \xi(t),
$$

where $M_{\sigma(t)}$ is a matrix satisfying $\mathscr{L}_{\sigma(t)}=M_{\sigma(t)} E$.

Note that $\xi(t)=0$ if and only if $x_{1}(t)=x_{2}(t)=\cdots=$ $x_{N}(t)$. Therefore, we will design appropriate feedback gain matrix and the topology switching law such that system (9) is global asymptotically stable. The averaging method will be used.

Lemma 8 (see [21]). Suppose there exists a constant $T$ for which the matrix-valued function $B(t)$ is such that $(1 / T) \int_{t}^{t+T} B(\tau) d \tau=\bar{B}$ for all t and

$$
\dot{x}(t)=(A(t)+\bar{B}) x(t), \quad x\left(t_{0}\right)=x_{0}, t \geq t_{0}
$$

is uniformly exponentially stable. Then, there exists $\varepsilon^{*}>0$ such that, for all fixed $\varepsilon \in\left(0, \varepsilon^{*}\right)$,

$$
\dot{x}(t)=\left(A(t)+B\left(\frac{t}{\varepsilon}\right)\right) x(t), \quad t \geq t_{0}
$$

is uniformly exponentially stable.

Let $\bar{M}=(1 / T) \int_{t}^{t+T} M_{\sigma(s)} d s=\sum_{s=t}^{t+T} \tau_{\sigma(s)} M_{\sigma(s)}$, where $\tau_{\sigma(s)}=\left(t_{k}^{j+1}-t_{k}^{j}\right) / T, j=k_{1}, \ldots, k_{m_{k}}, t_{k}^{k_{1}}=t$, and $t_{k}^{k_{m_{k}}}=t+T$. Considering Lemma 5, one has $\overline{\mathscr{L}}^{\text {av }}=$ $(1 / T) \int_{t}^{t+T} M_{\sigma(\tau)} E d \tau=\bar{M} E$. Since Assumption 3 holds, $\overline{\mathscr{L}}^{\mathrm{av}}$ has the property that $\operatorname{Re}(\lambda(E \bar{M}))>0$. Thus, from (9), one can obtain the time-average system

$$
\dot{\xi}(t)=\left(I_{N-1} \otimes(A+\Delta A)-c \bar{M} \otimes B K\right) \xi(t) .
$$

Theorem 9. Suppose that Assumption 3 holds; there exist a real scalar $c>0$ and a positive definite matrix $P>0$ such that

$$
\left[\begin{array}{ccc}
A^{T} P+P A-c \alpha P B B^{T} P & H^{T} & P D \\
H & -\frac{1}{\rho^{2}} I & 0 \\
D^{T} P & 0 & -I
\end{array}\right]<0,
$$


where $\alpha$ is defined in Lemma 7 satisfying $0<\alpha<$ $2 \min \{\operatorname{Re}(\lambda(E \bar{M}))\}$. Then, there exists a positive constant $\varepsilon^{*}>$ 0 such that the robust consensus of agents with the closed-loop dynamics

$$
\dot{x}(t)=\left(I_{N} \otimes(A+\Delta A)-c \mathscr{L}_{(t / \varepsilon)} \otimes B_{2} K\right) x(t)
$$

can be achieved for any $\varepsilon \in\left(0, \varepsilon^{*}\right)$, where the parameter $\varepsilon$ characterizes the speed of variation in $\mathscr{G}_{(t / \varepsilon)}$ and the feedback gain matrix can be designed as $K=B^{T} P$.

Proof. According to Schur complement lemma [22], linear matrix inequality (LMI) (13) holds if and only if the following inequality holds:

$$
A^{T} P+P A-c \alpha P B B^{T} P+\rho^{2} H^{T} H+P D D^{T} P<0 .
$$

Consider the following Lyapunov candidate of timeaverage system (12):

$$
V(t)=\xi(t)^{T}(Q \otimes P) \xi(t),
$$

where $P$ is a solution of inequality (13) and $Q$ is a feasible solution of (8). Then, the derivation of this Lyapunov candidate along the trajectory of system (12) is

$$
\begin{aligned}
\dot{V}(t)= & 2 \xi(t)^{T}\left(I_{N-1} \otimes(A+\Delta A)-c E \bar{M} \otimes B K\right)^{T} \\
& \cdot(Q \otimes P) \xi(t) .
\end{aligned}
$$

Substituting $K=B^{T} P$ into (17) yields

$$
\begin{aligned}
& \dot{V}(t)=\xi(t)^{T}\left(Q \otimes\left(A^{T} P+P A\right)\right. \\
& \left.-c\left((E \bar{M})^{T} Q+Q E \bar{M}\right) \otimes P B B^{T} P\right) \xi(t)+2 \xi(t)^{T} \\
& \cdot\left(Q \otimes(\Delta A)^{T} P\right) \xi(t)
\end{aligned}
$$

In light of the fact that $(E \bar{M})^{T} Q+Q E \bar{M}>\alpha Q$, where $0<\alpha<$ $2 \min \{\operatorname{Re}(\lambda(E \bar{M}))\}$, one has

$$
\begin{aligned}
\dot{V}(t) \leq & \xi(t)^{T}\left(Q \otimes\left(A^{T} P+P A-c \alpha P B B^{T} P\right)\right) \xi(t) \\
& +2 \xi(t)^{T}\left(Q \otimes(\Delta A)^{T} P\right) \xi(t) .
\end{aligned}
$$

Using Lemma 4, one has

$$
\begin{aligned}
2 \xi(t)^{T}\left(Q \otimes(\Delta A)^{T} P\right) \xi(t) & \\
\leq & \xi(t)^{T}\left(Q \otimes H^{T} F^{T}\right)\left(Q^{-1} \otimes I\right)(Q \otimes F H) \xi(t)^{T} \\
& +\xi(t)^{T}\left(I_{N-1} \otimes P D\right)(Q \otimes I)\left(I_{N-1} \otimes D^{T} P\right) \xi(t) \\
\leq & \xi(t)^{T}\left(Q \otimes H^{T} F^{T} F H\right) \xi(t)^{T} \\
& +\xi(t)^{T}\left(Q \otimes P D D^{T} P\right) \xi(t) \\
\leq & \xi(t)^{T}\left(Q \otimes\left(\rho^{2} H^{T} H+P D D^{T} P\right)\right) \xi(t) .
\end{aligned}
$$

In light of (20), it then follows from (19) that

$$
\begin{aligned}
& \dot{V}(t) \leq \xi(t)^{T}(Q \\
& \left.\otimes\left(A^{T} P+P A-c \alpha P B B^{T} P+\rho^{2} H^{T} H+P D D^{T} P\right)\right) \\
& \quad \xi(t) .
\end{aligned}
$$

Then, (15) implies that $\dot{V}(t)<0$. Thus, time-average system (12) is asymptotically stable.

According to Lemma 8, this means that there exists a positive constant $\varepsilon^{*}>0$ such that for all fixed $\varepsilon \in\left(0, \varepsilon^{*}\right)$

$$
\dot{\xi}(t)=\left(I_{N-1} \otimes(A+\Delta A)-c E M_{(t / \varepsilon)} \otimes B K\right) \xi(t)
$$

is asymptotically stable. That implies that if the topologies switch sufficiently fast, the consensus of system (14) will be achieved.

Remark 10. Commonly, the leader-following consensus problem can be conveniently converted into a stabilization problem by constructing the tracking error variables [14-17]. In contrast, relying on Lemma 5 and the variable transformation, the leaderless consensus problem of system (4) with directed fast switching topologies is successfully converted into the stabilization problem of fast switching system (9) here. Then, the averaging method is used to obtain the sufficient conditions for achieving consensus.

Remark 11. Most of existing works about the consensus problem of higher order systems with jointly connected topologies are restricted to be undirected topologies [10, 1418]. In this case, the graph Laplacian matrices are positive semidefinite. This property facilitated the construction of the Lyapunov function greatly in the derivation in both the leaderless and leader-following consensus problems. However, the Laplacian matrices of the directed graphs are usually not symmetric. Thus, the leaderless consensus problem with directed switching topologies is more challenging. Here, sufficient conditions for achieving consensus are obtained under directed switching topologies. It is just required that the union graph jointly contains a directed spanning tree, which means that the topologies are allowed to be unconnected in the switching time intervals. Although the topology condition in Assumption 3 is a bit strong, in which the union graph $\overline{\mathscr{G}}$ is assumed to be the same in each time interval $\left[t_{k}, t_{k+1}\right), k=0,1, \ldots$, a forward step has been made here towards a weaker communication topology condition that the union graph $\overline{\mathscr{G}}$ can be different in such time intervals.

\section{Examples}

In this section, we provide an example to illustrate the effectiveness of the above theoretical results. Consider a 


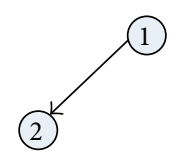

(4)

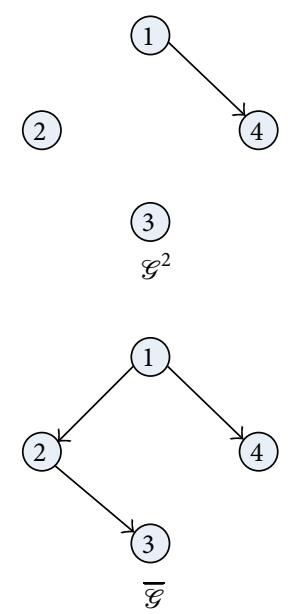

FIgURE 1: Communication topologies $\left\{\mathscr{G}^{1}, \mathscr{G}^{2}, \mathscr{G}^{3}\right\}$ and the union graph $\overline{\mathscr{G}}$.

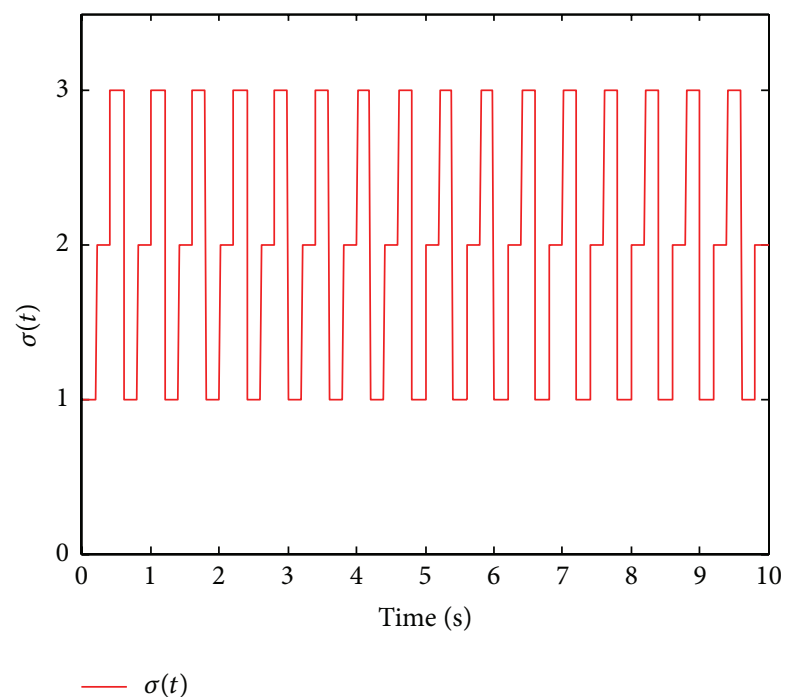

FIGURE 2: Switching signal.

multiagent system consisting of four agents in the form of position-speed model of moving plant with uncertainties

$$
\begin{gathered}
x_{i}=\left[\begin{array}{l}
x_{i 1} \\
x_{i 2}
\end{array}\right], \quad A=\left[\begin{array}{ll}
0 & 1 \\
0 & 0
\end{array}\right], \\
B=\left[\begin{array}{l}
0 \\
1
\end{array}\right], \quad D=H=\left[\begin{array}{ll}
1 & 0 \\
0 & 1
\end{array}\right],
\end{gathered}
$$

$F(t)=0.5 \sin (t)$. Then, $\rho=0.5$.

The directed switching communication topologies $\left\{\mathscr{G}^{1}, \mathscr{G}^{2}, \mathscr{G}^{3}\right\}$ are given in Figure 1. Clearly, the topologies are not connected and the union graph $\overline{\mathscr{G}}$ of $\left\{\mathscr{G}^{1}, \mathscr{G}^{2}, \mathscr{G}^{3}\right\}$ contains a directed spanning tree. The topologies are switching as $\mathscr{G}^{1} \rightarrow \mathscr{G}^{2} \rightarrow \mathscr{G}^{3} \rightarrow \mathscr{G}^{1} \rightarrow \cdots$ and each graph is active for 0.2 seconds. We can see that, for each $T=0.6$ seconds, $\overline{\mathscr{G}}$ contains a directed spanning tree.

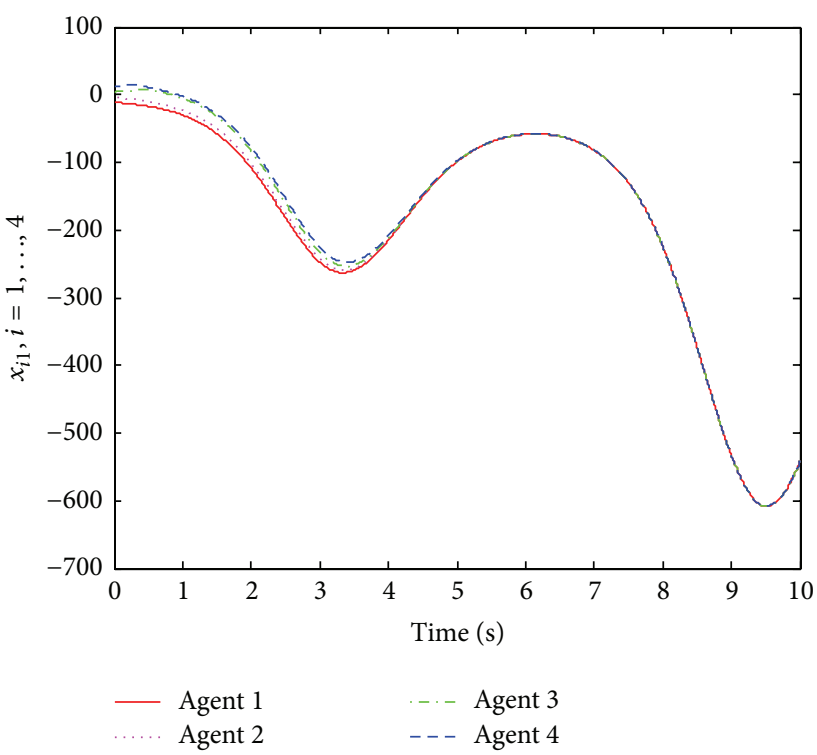

FIGURE 3: Trajectories of $x_{i 1}, i=1, \ldots, 4$.

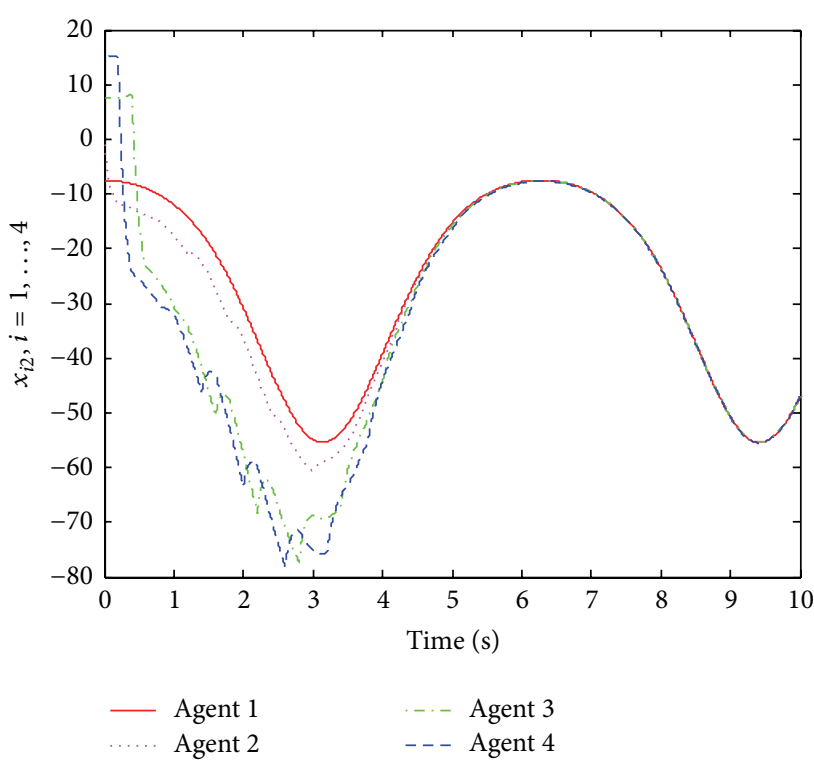

Figure 4: Trajectories of $x_{i 2}, i=1, \ldots, 4$.

Solving LMI (13) with $c=1.2, \alpha=1.5$, and $\rho=0.5$, one has

$$
P=\left[\begin{array}{cc}
5.4872 & 7.1563 \\
7.1563 & 10.5219
\end{array}\right]
$$

Then, the feedback matrix can be chosen as $K=$ [7.1563 10.5219]. Figures 3 and 4 show the states trajectories of all agents with the switching signal being shown in Figure 2. It is shown that the consensus is achieved. 


\section{Conclusions}

This paper has used the averaging method to solve the robust leaderless consensus problem of uncertain multiagent systems with fast switching topologies. The communication topologies are assumed to jointly contain a directed spanning tree. It has been proved that if the topologies switch sufficiently fast and the feedback matrix is properly designed, the consensus can be achieved even when the topologies are not connected in the switching time intervals.

\section{Conflict of Interests}

The authors declare that there is no conflict of interests regarding the publication of this paper.

\section{References}

[1] J. A. Fax and R. M. Murray, "Information flow and cooperative control of vehicle formations," IEEE Transactions on Automatic Control, vol. 49, no. 9, pp. 1465-1476, 2004.

[2] A. Jadbabaie, J. Lin, and A. S. Morse, "Coordination of groups of mobile autonomous agents using nearest neighbor rules," IEEE Transactions on Automatic Control, vol. 48, no. 6, pp. 988-1001, 2003.

[3] H. Chen, C. Wang, and Y. J. Lou, "Flocking multiple microparticles with automatically controlled optical tweezers: solutions and experiments," IEEE Transactions on Biomedical Engineering, vol. 60, no. 6, pp. 1518-1527, 2013.

[4] W. Ren and R. W. Beard, "Consensus seeking in multiagent systems under dynamically changing interaction topologies," IEEE Transactions on Automatic Control, vol. 50, no. 5, pp. 655661, 2005.

[5] Y. Fan, L. Liu, G. Feng, C. Song, and Y. Wang, "Virtual neighbor based connectivity preserving of multi-agent systems with bounded control inputs in the presence of unreliable communication links," Automatica, vol. 49, no. 5, pp. 1261-1267, 2013.

[6] K. Chen, J. Wang, Y. Zhang, and Z. Liu, "Second-order consensus of nonlinear multi-agent systems with restricted switching topology and time delay," Nonlinear Dynamics, vol. 78, no. 2, pp. 881-887, 2014.

[7] H. Li, X. Liao, X. Lei, T. Huang, and W. Zhu, "Secondorder consensus seeking in multi-agent systems with nonlinear dynamics over random switching directed networks," IEEE Transactions on Circuits and Systems I: Regular Papers, vol. 60, no. 6, pp. 1595-1607, 2013.

[8] M. J. Park, O. M. Kwon, J. H. Park, S. M. Lee, and E. J. Cha, "Leader following consensus criteria for multi-agent systems with time-varying delays and switching interconnection topologies," Chinese Physics B, vol. 21, no. 11, Article ID 110508, 2012.

[9] Y.-J. Sun, G.-L. Zhang, S.-X. Zhang, and J. Zeng, "Consensus analysis for a class of heterogeneous multi-agent systems in fixed and switching topology," Acta Physica Sinica, vol. 63, no. 22, Article ID 220201, 2014.

[10] Y. Su and J. Huang, "Stability of a class of linear switching systems with applications to two consensus problems," IEEE Transactions on Automatic Control, vol. 57, no. 6, pp. 1420-1430, 2012.
[11] G. Wen, W. Yu, J. Cao, G. Hu, and G. Chen, "Consensus control of switching directed networks with general linear node dynamics," in Proceedings of the 9th Asian Control Conference (ASCC '13), pp. 1-6, IEEE, Istanbul, Turkey, June 2013.

[12] G. Wen, G. Hu, W. Yu, J. Cao, and G. Chen, "Consensus tracking for higher-order multi-agent systems with switching directed topologies and occasionally missing control inputs," Systems \& Control Letters, vol. 62, no. 12, pp. 1151-1158, 2013.

[13] G. Wen and V. Ugrinovskii, "Distributed consensus of linear multi-agent systems with switching directedtopologies," http://arxiv.org/abs/1409.5519.

[14] W. Ni, X. Wang, and C. Xiong, "Consensus controllability, observability and robust design for leader-following linear multi-agent systems," Automatica, vol. 49, no. 7, pp. 2199-2205, 2013.

[15] W. Ni, X. Wang, and C. Xiong, "Leader-following consensus of multiple linear systems under switching topologies: an averaging method," Kybernetika, vol. 48, no. 6, pp. 1194-1210, 2012.

[16] W. Xu, J. Cao, W. Yu, and J. Lu, "Leader-following consensus of non-linear multi-agent systems with jointly connected topology," IET Control Theory \& Applications, vol. 8, no. 6, pp. 432440, 2014.

[17] X. Mu, X. Xiao, K. Liu, and J. Zhang, "Leader-following consensus of multi-agent systems with jointly connected topology using distributed adaptive protocols," Journal of the Franklin Institute, vol. 351, no. 12, pp. 5399-5410, 2014.

[18] Q. Jia and W. K. S. Tang, "Averaging method for consensus of fast switching multi-agent networks with communication delay," in Proceedings of the 26th Chinese Control and Decision Conference (CCDC '14), pp. 258-262, Changsha, China, June 2014.

[19] R. A. Horn and C. R. Johnson, Matrix Analysis, Cambridge University Press, New York, NY, USA, 1985.

[20] S. Zhou, W. Liu, Q. Wu, and G. Yin, "Leaderless consensus of linear multi-agent systems: matrix decomposition approach," in Proceedings of the 7th International Conference on Intelligent Human-Machine Systems and Cybernetics, pp. 327-332, Hangzhou, China, August 2015.

[21] D. J. Stilwell, E. M. Bollt, and D. G. Roberson, "Sufficient conditions for fast switching synchronization in time-varying network topologies," SIAM Journal on Applied Dynamical Systems, vol. 5, no. 1, pp. 140-156, 2006.

[22] S. Boyd, L. El Ghaoui, E. Feron, and V. Balakrishnan, Linear Matrix Inequalities in Systems and Control Theory, SIAM, Philadelphia, Pa, USA, 1994. 


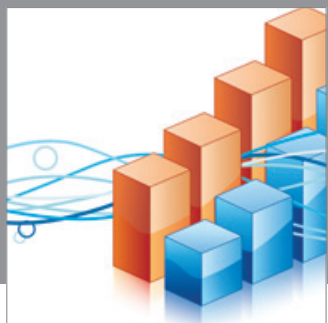

Advances in

Operations Research

mansans

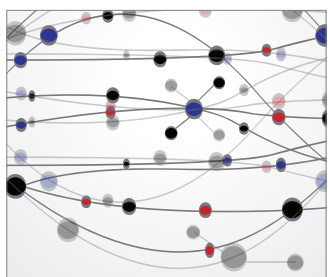

The Scientific World Journal
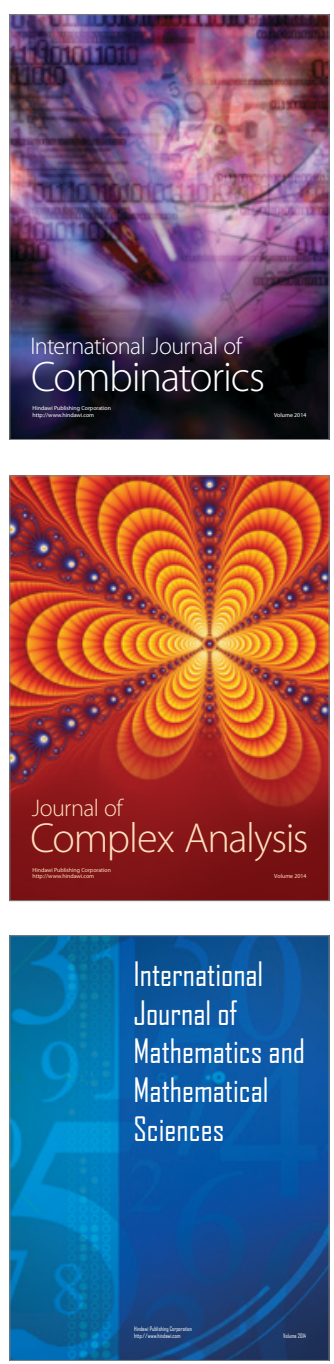
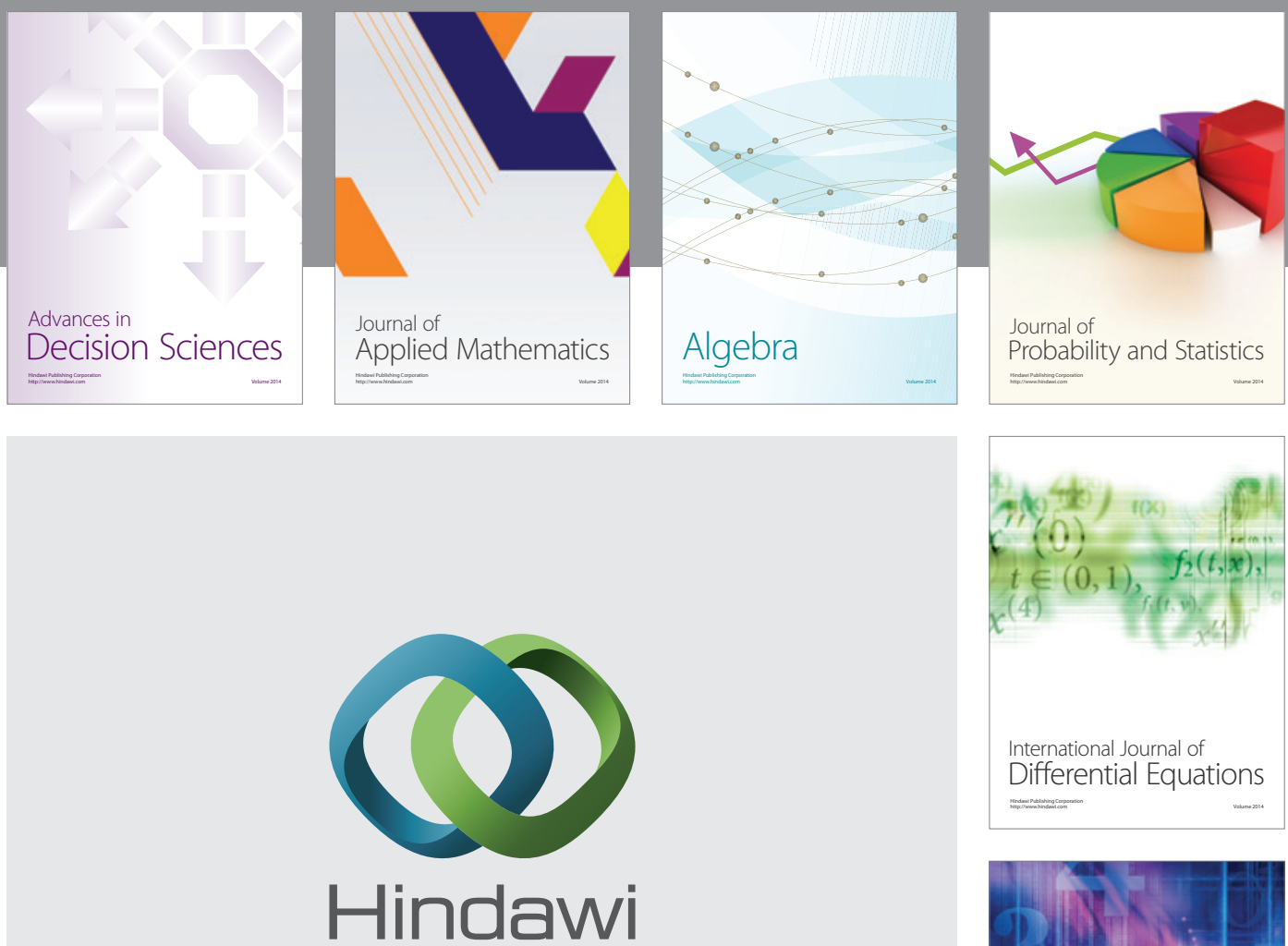

Submit your manuscripts at http://www.hindawi.com
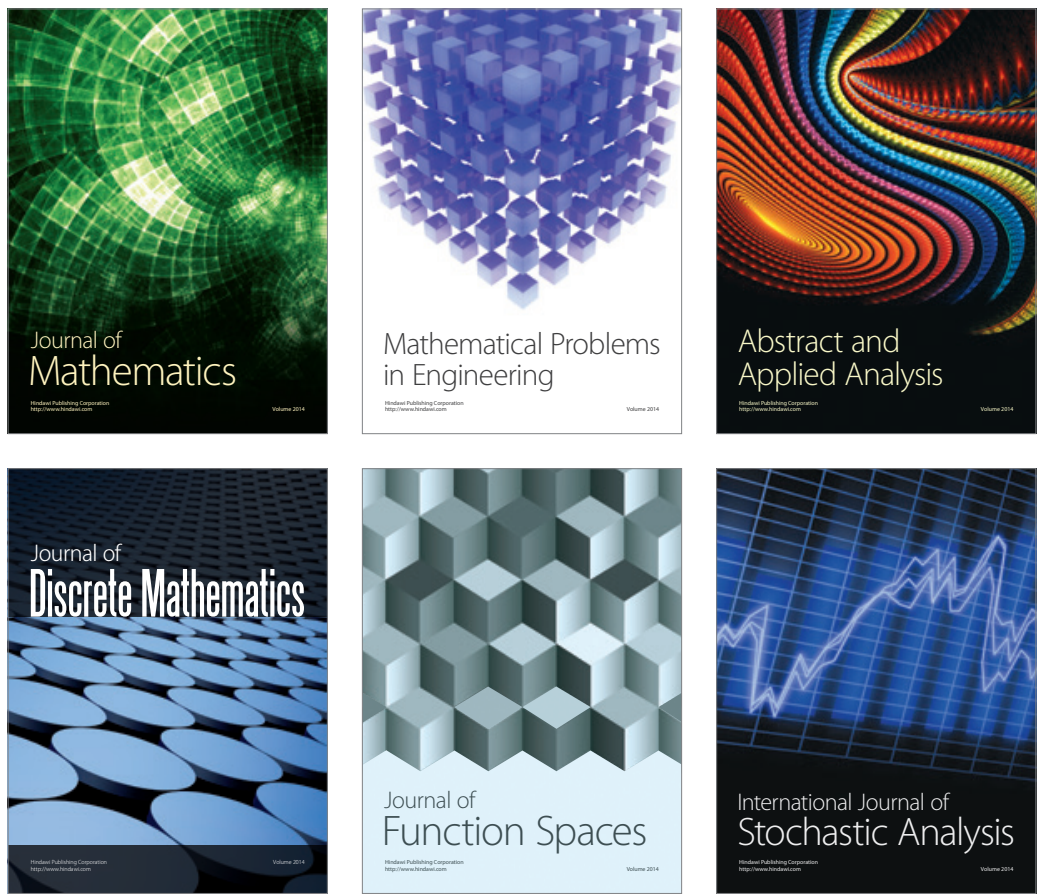

Journal of

Function Spaces

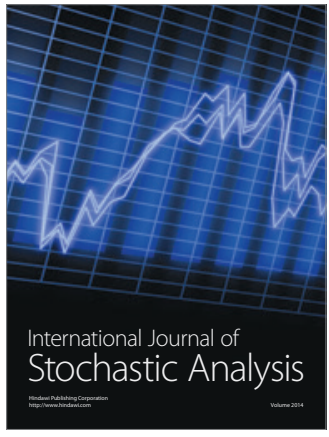

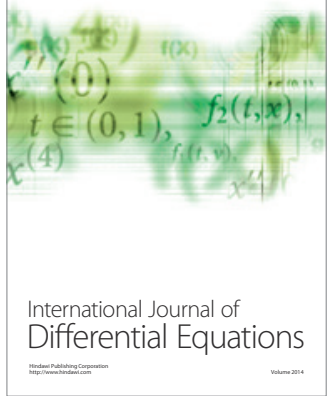
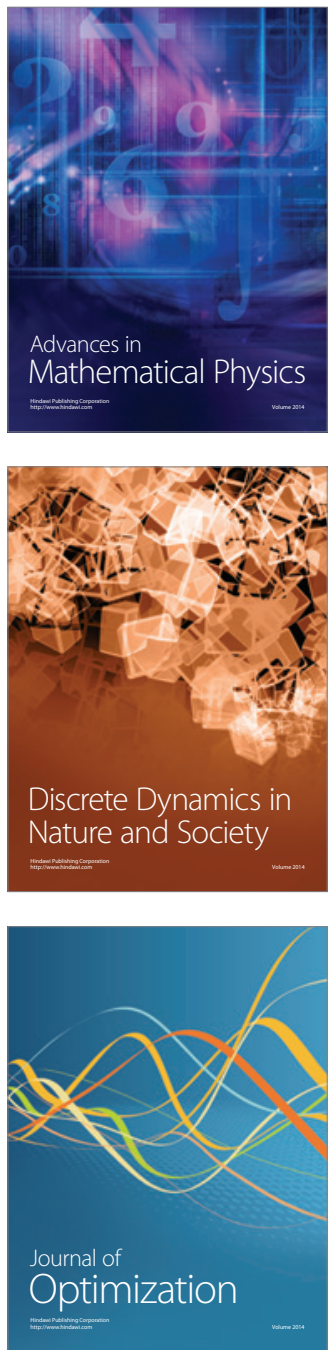